\title{
DEFLEGER: DEslocamento e FLExibilidade de cargas no Gerenciamento de Energia Residencial
}

\author{
Tiago B. Castro ${ }^{1}$, Natalia C. Fernandes ${ }^{1}$, Marcio Z. Fortes ${ }^{1}$ \\ ${ }^{1}$ Programa de Pós-Graduação em Engenharia Elétrica e de Telecomunicações (PPGEET) \\ Universidade Federal Fluminense (UFF) \\ Niterói - RJ - Brasil \\ \{tiago_bornia, nataliacf\}@id.uff.br, mzf@vm.uff.br
}

\begin{abstract}
The maintenance of the balance between energy production and consumption is essential for the power grid stability. With the implementation of smart grids, there is a paradigm change: in traditional power grid, production adapts to the demand, but in smart grids, demand adapts to the production and makes user consumption more efficient. Demand Side Management (DSM) programs arise as one of the solutions to adjust user consumption to supply. The purpose of this work is the creation of DSM mechanisms for Brazilian users, using IoT and smart home concepts. DEFLEGER-ECO was created as an mechanism for saving energy using variable tariffs and DEFLEGER-EMER as an emergency mechanism aimed at reducing demand during emergency events. The models were simulated in four residential profiles in order to evaluate their performance. The results show that the proposed mechanisms are efficient and help to reduce the demand according to the consumption goals of the user and also in critical moments of the power system.
\end{abstract}

Resumo. A manutenção do equilíbrio entre a produção e o consumo de energia é essencial para a estabilidade da rede elétrica. Com a implantação das smart grids, há uma mudança de paradigma: na rede tradicional, a produção adaptase à demanda, mas nas smart grids, a demanda adapta-se à produção e faz com que o consumo de usuário seja mais eficiente. Os programas de Gerenciamento pelo Lado da Demanda (GLD) surgem como uma solução para ajustar o consumo do usuário à geração. A proposta deste trabalho é a criação de mecanismos de GLD direcionado para o usuário residencial no Brasil, utilizando os conceitos de IoT e de casas inteligentes. Foi criado o DEFLEGER-ECO, mecanismo de economia de energia elétrica usando tarifa variável, e o DEFLEGEREMER, mecanismo emergencial voltado a redução de demanda durante eventos emergenciais. Os modelos foram simulados em quatro perfis de residências com a finalidade de avaliar seus desempenhos. Os resultados dos testes mostram que os mecanismos são eficientes e ajudam a reduzir a demanda conforme as metas de consumo do usuário e também em eventos críticos do sistema de potência.

\section{Introdução}

Smart grids surgem como a evolução da rede elétrica. Sua proposta é incorporar redes de telecomunicações à rede elétrica, possibilitando o monitoramento em tempo real, rápida detecção/tratamento de falhas, maior facilidade de integração de fontes renováveis entre outros serviços. 
Com sua implantação, há comunicação bidirecional entre a empresa de energia e as unidades consumidoras. Isto se torna possível através da instalação de medidores inteligentes nas unidades consumidoras. Surge também a infraestrutura avançada de medição, um sistema que conecta o operador de distribuição com o cliente. Desta forma, a concessionária tem informação em tempo real do comportamento de consumo dos usuários e os usuários têm informação detalhada dos seus consumos.

Entretanto, com o aumento do número de fontes renováveis na rede, a predição da energia produzida torna-se mais difícil, devido à natureza intermitente dessas fontes. Fontes de energia renováveis dependem de fatores ambientais que tornam a previsão de geração de energia mais complexa e menos precisa. Isso afeta diretamente a manutenção do equilíbrio entre a produção e o consumo de energia, que é essencial para a estabilidade da rede elétrica. Portanto, existe uma mudança de paradigma com a modernização de rede de energia: na rede tradicional, a produção adapta-se à demanda, mas nas smart grids, a demanda adapta-se à produção e faz com que o consumo de usuário seja mais eficiente.

Os programas de Gerenciamento pelo Lado da Demanda (GLD) surgem como uma solução para ajustar o consumo do usuário à geração. GLD são ações ou decisões tomadas pela empresa de energia para alterar ou modelar o padrão de consumo do usuário. No Brasil, existe aplicação do GLD no setor industrial, porém sua aplicação no setor residencial começa a ser estudada devido a regulamentação da Tarifa Branca [ANEEL-RN733 2016], tarifa dinâmica que está sendo implantado no Brasil. Ela é melhor explicada na Seção 2.

Cada unidade consumidora residencial causa um impacto muito pequeno na demanda total de energia da rede, porém, somadas, elas correspondem à 9,9\% do consumo nacional, segundo o Balanço Energético Nacional de 2019 [EPE 2019]. Então, para que haja um impacto relevante, os programas de GLD devem alcançar um grande número de clientes. Por isso, é preciso desenvolver modelos flexíveis, que atendam diversos perfis de clientes de energia elétrica. Apesar do setor residencial corresponder à apenas 9,9\% do consumo nacional, é ele que aumenta o custo marginal de manutenção e expansão da rede de distribuição elétrica, por não apresentar políticas de controle para o horário de ponta, assim como os demais setores [EPE 2016].

Neste trabalho, foram criados dois mecanismos de GLD baseados em computação urbana. O DEFLEGER-ECO, mecanismo de economia de energia elétrica usando Tarifa Branca, e DEFLEGER-EMER, mecanismo emergencial voltado a redução de demanda durante eventos emergenciais. O mecanismo DEFLEGER-ECO cria uma gerência automática do consumo de energia residencial, de forma a auxiliar o usuário a economizar energia elétrica no horário de pico. Já o DEFLEGER-EMER, visa a manutenção da sanidade do fornecimento de energia em uma região do país diante de eventos críticos (que necessitem redução de demanda). Nesse sentido, quando há um evento crítico, um aviso de emergência é enviado às residências, que reduzem automaticamente o consumo de acordo com as prioridades dos usuários.

Muitas das propostas de GLD até o momento realizam apenas deslocamento de cargas em horário de pico, ou seja, reagendam o funcionamento de alguns dispositivos do horário de pico para um horário fora de pico. Outras propostas utilizam apenas o ajuste do termostato de aparelhos de ar-condicionado ou aquecedores para economizar 
energia [Gelazanskas and Gamage 2014] [Li and Zhang 2014]. Os modelos propostos neste trabalho utilizam o deslocamento de carga, o ajuste de termostato e ainda fazem um ajuste de potência de dispositivos ajustáveis. Estes dispositivos ajustáveis são aparelhos que podem operar em diferentes potências, por exemplo ventiladores (ao funcionar em diferentes velocidades) ou até mesmo lâmpadas inteligentes. Assim, com os mecanismos propostos, é possível criar programas GLD residenciais com maior possibilidade de implantação em larga escala, sem se restringirem aos usuários com ar-condicionado. Para avaliar o sistema, foi utilizado um simulador que considera o consumo de energia e a comunicação entre os dispositivos inteligentes. Ambos os mecanismos DEFLEGERECO e DEFLEGER-EMER foram avaliados, mostrando sua eficiência no atendimento das metas apresentadas.

O restante do artigo está organizado como descrito a seguir. A Seção 2 apresenta uma contextualização sobre o tema, enquanto que a Seção 3 descreve os principais trabalhos relacionados. A Seção 4 apresenta a proposta de programas de GLD. A Seção 5 descreve o ambiente de simulação e a Seção 6 traz a análise dos resultados. Por fim, a Seção 7 conclui o trabalho.

\section{Contextualização}

Em uma casa inteligente (smart home), assume-se que os dispositivos são inteligentes, possuindo uma interface de comunicação que permite que o comportamento destes equipamentos possa ser monitorado e alterado remotamente.

Os mecanismos de GLD propostos são aplicados em smart homes, baseados na comunicação com os dispositivos gerenciáveis e com o smart meter, que periodicamente reporta ao usuário o consumo de energia, tanto da geração própria (quando na residência existir micro ou mini geração distribuída), quanto da energia fornecida pela concessionária de energia elétrica local. Esse tipo de sistema permite, entre outras funcionalidades, que a smart grid envie informações como preços dinâmicos da tarifa energia condicionada ao horário de uso [Komninos et al. 2014]. A Figura 1 apresenta a arquitetura de rede de uma smart home.

A smart home é dividida em duas áreas: a área externa e a área interna. O limite da área interna é o sistema de gerenciamento de energia (Energy Management System - EMS), que se comunica com as cargas internas e faz o gerenciamento delas. Periodicamente, os dispositivos enviam informações de consumo para o EMS, que usa essas informações para alimentar seus algoritmos de funcionamento. O mecanismo GLD é gerenciado pelo EMS.

O limite da área externa é feito por dois dispositivos: o smart meter e a Interface de Serviço de Energia (Energy Services Interface - ESI). Eles funcionam como gateways e fazem a troca de dados entre o domínio do consumidor e os domínios externos. O smart meter foi projetado para tratar apenas os dados agregados de medição [Lee et al. 2013]. Já o ESI dá suporte ao controle remoto de serviços, aos programas de resposta à demanda, ao monitoramento de fontes de energia renováveis e ao monitoramento de veículos elétricos. Apesar de desempenharem funções diferentes, o ESI e os smart meters podem ser fisicamente integrados em um mesmo dispositivo [Komninos et al. 2014].

Os mecanismos de GLD são definidos como o planejamento, implantação e monitoramento das atividades da concessionária de energia, que objetivam influenciar o com- 


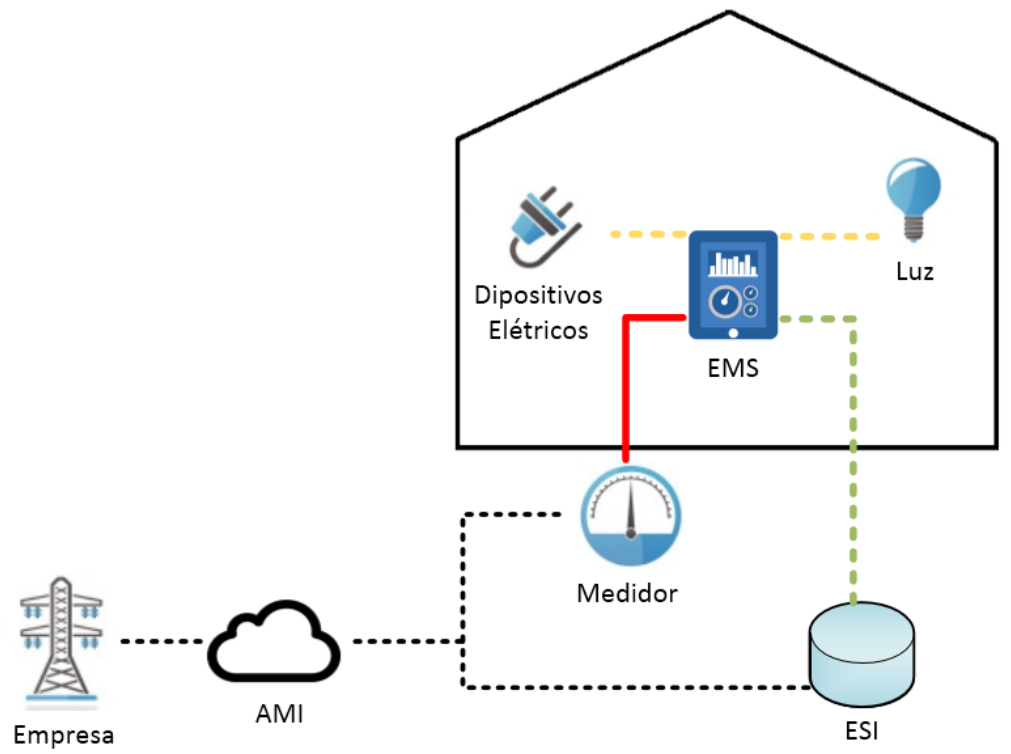

Figura 1. Arquitetura de uma smart home adaptada de [Komninos et al. 2014].

portamento do usuário em relação ao consumo elétrico [Gelazanskas and Gamage 2014]. Estas influências visam, principalmente, alterar o padrão de consumo achatando a curva dos horários de pico, ou até mesmo modelar a curva de consumo conforme a produção energética. São utilizadas técnicas de modelagem de carga, como deslocamento de carga, reagendamento para um horário mais oportuno, ou flexibilidade, por exemplo ao diminuir a carga através da alteração de termostato.

Uma outra forma de realizar a GLD é pela alteração das tarifas de energia. No Brasil, o padrão de cobrança residencial com tarifas variáveis é chamado de Tarifa Branca [ANEEL-RN733 2016]. Nessa forma de tarifação o preço do kWh varia ao longo do dia buscando refletir o custo da geração de energia em cada momento. Nos horários de pico o preço do $\mathrm{kWh}$ é bem elevado, se comparado à tarifa convencional, e fora do horário de pico o preço do kwh é menor. Existem três preços diferentes para kWh ao longo do dia, a Figura 2 apresenta um comparativo entre a tarifa convencional e a Tarifa Branca.

\section{Trabalhos Relacionados}

Diversas iniciativas vêm sendo propostas de forma a viabilizar a aplicação em larga escala da GLD, de forma a reduzir os custos para as empresas distribuidoras e para os clientes do sistema de potência.

Kinhekar et al. incentivam o uso de tarifação dinâmica na Índia como um alternativa para redução de custo operacional e propõem um mecanismo GLD baseado no deslocamento de carga do horário de pico para horários de menor demanda [Kinhekar et al. 2013]. São estabelecidos os preços ao longo do dia e uma curva de consumo desejável é calculada, sendo ela inversamente proporcional à curva de preços. $\mathrm{O}$ deslocamento de cargas é usado para reagendar o funcionamento de alguns equipamentos, de maneira que o a curva de consumo final seja bem próxima da curva desejada.

Conejo et al. descrevem um modelo de otimização para ajustar o nível de carga de um determinado consumidor em resposta aos preços de eletricidade por 


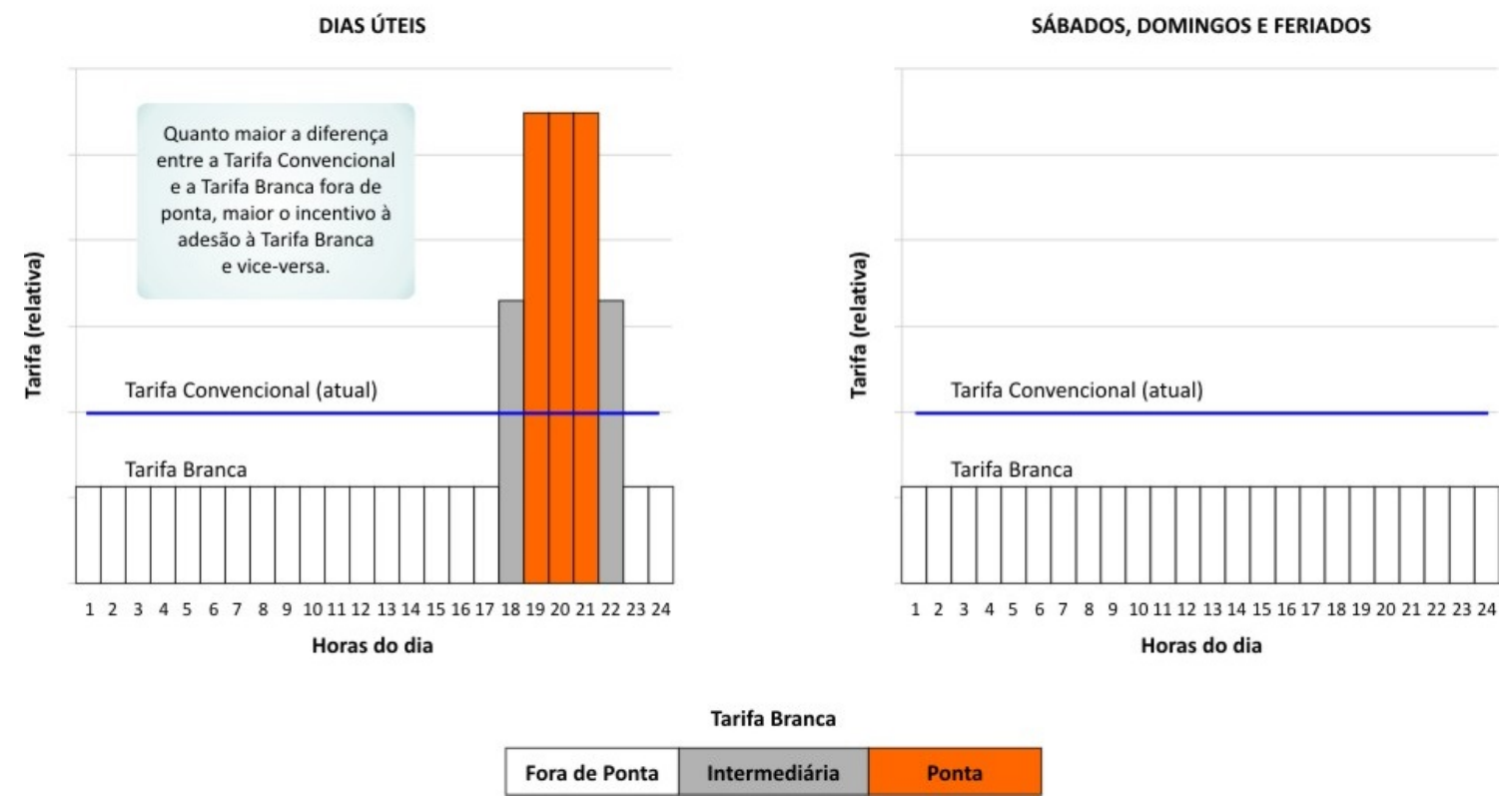

Figura 2. Tarifa Branca [ANEEL 2020].

hora [Conejo et al. 2010]. O sistema utiliza uma previsão da variação dos preços para traçar um plano de consumo através de um algoritmo linear de programação. A cada hora, a concessionária envia o preço real e o algoritmo é realimentado, traçando, assim, um novo plano de consumo para o restante do dia e atualizando o algoritmo de previsão.

Tham e Zhou propõe um programa baseado em acúmulo de pontos e sensoriamento ambiente, onde a ideia é a penalização pelo mal uso de energia e a bonificação pelo uso correto [Tham and Zhou 2013]. A temperatura e umidade relativa do ar são coletadas e usadas em um cálculo para estabelecer se aquele é um dia quente ou um dia frio. Se em um dia quente o usuário tomar banho quente, ele perderá pontos, mas, se em um dia frio, o usuário tomar banho frio, ele ganhará pontos. No final do mês, os pontos são convertidos em descontos ou em multas na conta de energia do consumidor.

Li e Zhang apresentam e comparam alguns modelos que trabalham com aparelhos de climatização ambiente, podendo ser classificados como Heating, Venting and Cooling (HVAC) [Li and Zhang 2014]. A ideia desses mecanismos é alteração do termostato dos aparelhos de acordo com a variação de preço da energia. Geralmente, são modelos muito eficazes, onde se consegue grande economia com a variação de alguns graus da temperatura ambiente.

Estas soluções trabalham somente com deslocamento de cargas do horário de pico ou somente com o ajuste da temperatura de um determinado dispositivo elétrico, condicionado à variação de preço. Este trabalho propõe dois mecanismos residenciais que combinam tanto o deslocamento de carga como a variação de potência de alguns dispositivos (sendo eles de manutenção da temperatura ambiente ou não). O primeiro mecanismo se trata de um modelo de economia de energia em horário de pico usando tarifação dinâmica. O segundo se trata de um modelo emergencial para redução de demanda durante eventos críticos (que necessitem redução de demanda) na rede elétrica. 


\section{Proposta de Programas GLD}

Nesse trabalho, são propostos dois mecanismos de GLD, sendo um baseado em tarifação dinâmica e o outro voltado para eventos emergenciais na rede. A ideia é que o usuário possa escolher entre o mecanismo que ele se adapte melhor, mas nada impede que ele escolha os dois. Os modelos fazem uma gestão automática dos dispositivos elétricos da casa buscando economizar durante o horário de ponta da Tarifa Branca ou atender ao pedido de redução de demanda durante eventos emergencias da rede elétrica.

Os modelos utilizam duas técnicas de modelagem: deslocamento de carga e flexibilidade de carga. $\mathrm{O}$ deslocamento realiza um reagendamento da carga para um outro horário, e a flexibilidade consiste em ajustar a potência de uso de alguns dispositivos, por exemplo o aparelho de ar-condicionado que pode ter sua temperatura ajustada para economizar ou chuveiros inteligentes que podem trabalhar com vários níveis de potência. Conforme os parâmetros setados no programa, o usuário pode escolher se prefere utilizar deslocamento, flexibilidade ou as duas técnicas.

O modelo de economia é baseado no uso da Tarifa Branca. O usuário configura seus parâmetros de conforto e o percentual que deseja economizar durante o horário de ponta da Tarifa Branca. O mecanismo busca alcançar a meta de economia, entretanto respeitando os parâmetros de conforto configurados. Se ele não conseguir atingir o percentual de economia desejado, uma mensagem é enviada comunicando isto ao usuário.

O Modelo emergencial realiza redução de demanda ao receber uma mensagem da concessionária. Em momentos de emergência na rede elétrica, mensagens contendo o percentual de carga a ser reduzido são enviadas aos smart meters de uma determinada região. Ao receber a mensagem, o mecanismo atua sobre as cargas a fim de garantir que a meta de redução seja alcançada.

\subsection{Classificação de Cargas}

O funcionamento dos mecanismos propostos é baseado na divisão dos aparelhos da casa por grupos, onde cada grupo terá um tratamento diferente durante a etapa em que a meta dos programas é atingida.

Os dispositivos são divididos em quatro grupos:

- Reguláveis - qualquer dispositivo que possua potência ajustável. Por exemplo, ar-condicionado, lâmpadas inteligentes e chuveiros inteligentes;

- Flexíveis - qualquer dispositivo que o usuário classificar como deslocável, ou seja, com agendamento deslocável. Por exemplo, máquina de lavar roupas ou secadora de roupas;

- Inflexíveis dispensáveis (Dispensáveis) - dispositivos que não possuem potência ajustável, nem são classificados como deslocáveis, mas que a interrupção de funcionamento não afeta muito o usuário. Por exemplo, filtro de água;

- Inflexíveis indispensáveis (Indispensáveis) - dispositivos não agendáveis, sem potência ajustável, porém a interrupção causa grande impacto no conforto do usuário. Por exemplo, televisão e computador.

\subsection{DEGLEGER-ECO}

O primeiro modelo foi denominado de DEslocamento e FLExibilidade de cargas no Gerenciamento de Energia Residencial - ECOnomia (DEFLEGER-ECO). Este programa foi 
desenvolvido para auxiliar os usuários na economia de energia em horário de pico, principalmente aqueles que optarem pelo uso da tarifa branca. Através de deslocamento de cargas e da redução de potência de alguns dispositivos, ele busca atingir a um percentual de economia preestabelecido pelo cliente para o horário de ponta e intermediário. Como o objetivo deste mecanismo é tentar influenciar o padrão de consumo, os parâmetros de conforto dos clientes são respeitados.

O usuário classifica os dispositivos elétricos de sua casa, conforme a classificação apresentada na subseção anterior. O parâmetro Intensidade (seus valores variam entre 1 e 5) modela o nível de conforto do usuário. Se Intensidade for 1, o nível de potência dos dispositivos Reguláveis não podem ser alterados. Se a Intensidade configurada pelo usuário for 5, o mecanismo tem liberdade para variar a potência o máximo possível. Para valores intermediários, a potência oscila entre o mínimo e o máximo de variação.

Outro parâmetro que o cliente pode configurar é o percentual de economia que ele deseja alcançar nos horários de pico/intermediários. O cálculo da meta de economia é alcançado através da multiplicação da media histórica de demandas $\left(M e d_{\text {hist }}\right)$ daquele horário pelo percentual de economia $\left(P_{\text {eco }}\right)$ configurado pelo usuário para o horário de pico.

Periodicamente, o modelo verifica se o horário atual corresponde ao horário de pico ou intermediário. Se sim, o mecanismo de economia é acionado. A lógica do mecanismo emergencial é mostrada na Figura 3.

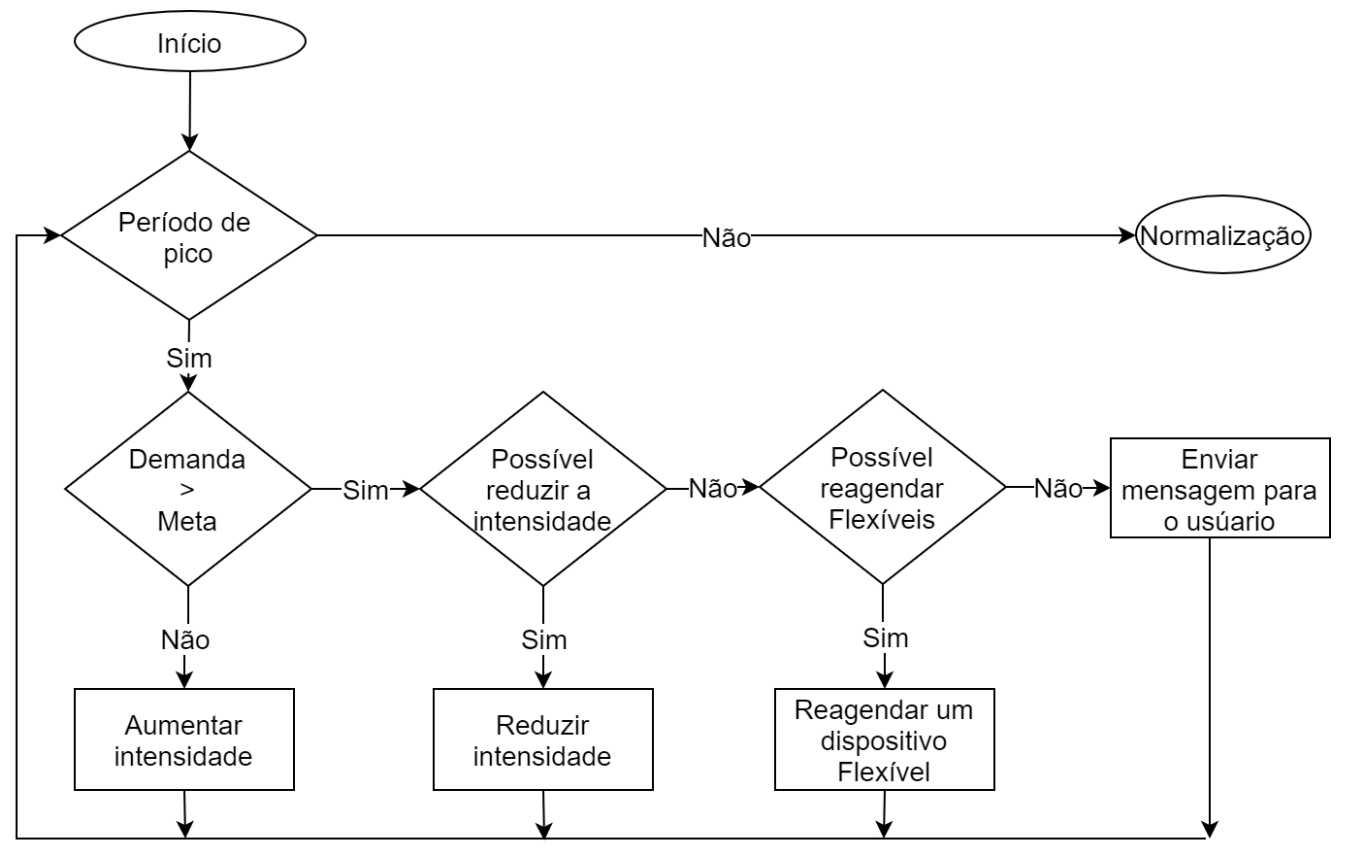

Figura 3. Fluxograma do funcionamento do DEFLEGER-ECO.

O algoritmo de economia busca de forma gradual alcançar a meta trabalhando com os grupos Reguláveis e Flexíveis. Primeiro, vai se reduzindo a potência dos Reguláveis até a demanda ser menor que a meta, ou até que todos os Reguláveis alcancem a potência mínima, respeitando os parâmetros de conforto configurados. Se a meta não for atingida, desliga-se os Flexíveis até atingir a meta. Caso a demanda continue acima do desejado, 
o usuário é sinalizado que a economia máxima, respeitando o conforto dele, já foi feita e que ele deve tomar alguma ação se quiser se manter dentro da meta. Nos casos em que a meta for alcançada, o mecanismo analisa se é possível religar algum aparelho ou aumentar a potência de algum dispositivo que tenha sido reduzida. Após o fim do período de ponto o DEFLEGER-ECO envia comandos normalizando o funcionamento de todos os dispositivos.

É importante notar que todo o controle é feito continuamente, com pequenos intervalos de tempo entre cada rodada, pois o consumo do usuário é variável e os ajustes precisam ser realizados de acordo com a ativação ou desativação das cargas pelo usuário. Além disso, alguns ajustes de intensidade demandam algum tempo para surtir efeito, como a temperatura do ar-condicionado, de forma que não seria eficiente executar ininterruptamente as rodadas.

\subsection{DEFLEGER-EMER}

O modelo emergencial foi denominado de DEslocamento e FLExibilidade de cargas no Gerenciamento de Energia Residencial - EMERgencial (DEFLEGER-EMER). Diferente do programa de economia, o programa DEFLEGER-EMER permite que a empresa de energia exerça uma influência mais direta no padrão de consumo do usuário. Em eventos emergenciais do sistema, a concessionária envia uma mensagem com um percentual de redução de carga para o cliente e ele deve obrigatoriamente atender a essa solicitação. Os eventos emergenciais podem ocorrer para evitar sobrecarga ou por qualquer outro motivo que a redução se fizer necessária, e a maneira como o usuário vai atender ao pedido é transparente à empresa de energia. Deve ser estabelecido contrato que regule quantidade máxima de intervenções e período máximo de tempo dessas intervenções. A participação nesse programa pode ser recompensada com descontos na fatura de energia. Nos modelos americanos [Clearlyenergy 2016], o desconto é proporcional à carga que é disponibilizada ao programa.

O estabelecimento dos parâmetros de conforto e a fase de classificação de cargas funcionam da mesma forma que no algoritmo DEFLEGER-ECO. A única diferença é que no mecanismo DEFLEGER-EMER se aplica uma nova lógica, que deve ser mais agressiva e assertiva. Nesse caso, ao receber uma mensagem da concessionária indicando um evento emergencial, o gerenciador deve garantir demanda abaixo de determinada meta. Assim, o cálculo da meta emergencial é alcançado com a multiplicação da demanda no início do evento emergencial $\left(D_{\text {atual }}\right.$ ) pelo percentual de redução recebido na mensagem de emergência $\left(P_{\text {emergencial }}\right)$. A lógica do mecanismo emergencial é mostrada na Figura 4.

$\mathrm{O}$ algoritmo emergencial busca alcançar a meta trabalhando com os grupos Reguláveis e Flexíveis, porém se for necessário ele também trabalha com os Inflexíveis. Quando a concessionária envia uma mensagem de emergência, o mecanismo de emergencial é acionado. Ele trabalha em duas fases. A primeira fase busca respeitar o conforto do usuário, mas a segunda fase não respeita os parâmetros do usuário e busca atingir a meta de forma rápida.

A fase inicial começa com a redução de potência dos Reguláveis para o mínimo. Aguarda-se, então, um período de tempo para estabilizar a demanda dos aparelhos de arcondicionado. Se a meta não for alcançada, agendam-se os Flexíveis. Se mesmo assim não houver sucesso começa-se a fase dois, os parelhos de ar-condicionado são desligados, 


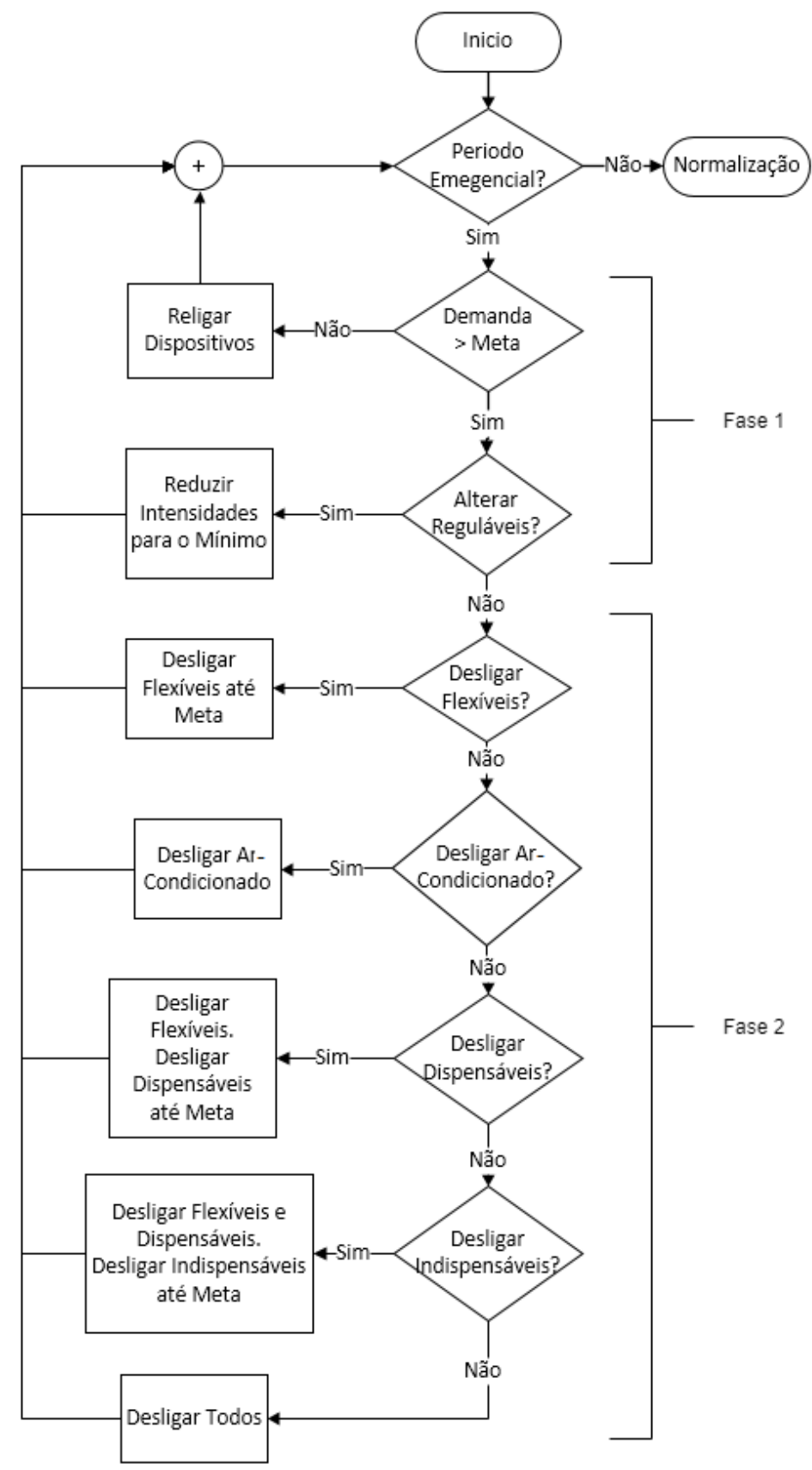

Figura 4. Fluxograma do funcionamento do DEFLEGER-EMER.

por apresentarem grande potência. Se a meta não tiver sido atingida, o próximo grupo a ser desligado é o dos Dispensáveis e depois os Indispensáveis. O algoritmo vai evoluindo e aumentando o número de grupos de ação. Caso necessário, todos os equipamentos gerenciáveis da casa podem ser desligados. Entretanto, a ideia é evitar ao máximo o desligamentos dos dispositivos Indispensáveis.

Após a meta ser alcançada, o algoritmo verifica se pode religar algum dispositivo sem ultrapassar a meta. Se o ar-condicionado for desligado ele apenas será religado após o fim do período emergencial. Quando a concessionário envia uma nova mensagem sinalizando o fim do período emergencial, o DEFLEGER-EMER envia comandos 
normalizando o funcionamento de todos os dispositivos, seja religando ou reajustando as potências de uso.

\section{Ambiente de Simulação}

Como o mecanismo proposto trata-se de um sistema de controle para sistemas elétricos domésticos, é necessário que seja possível implementar a lógica de controle, a resposta na rede elétrica e a troca de mensagens para monitoração e ativação dos comandos. Assim, deve-se simular não só a rede elétrica, mas também a rede de comunicação, além da lógica de controle proposta. Para isso foi utilizada a ferramenta de simulação desenvolvida e descrita por [Castro 2016], com os mesmos parâmetros usados por ele. Esta ferramenta consiste na integração do simulador de rede NS-3 com o simulador de consumo de energia EnergyPlus.

\subsection{Cenários}

Para simular diferentes perfis de consumo residencial, foram utilizados quatro perfis de usuários adaptados do projeto Casa Eficiente [Lamberts et al. 2010a]:

- Perfil 1 - "Família Sbanja" com equipamentos ineficientes;

- Perfil 2 - "Família Sbanja" com equipamentos eficientes;

- Perfil 3 - "Família Consciente" com equipamentos ineficientes;

- Perfil 4 - "Família Consciente" com equipamentos eficientes.

Os perfis de "Família Sbanja"possuem níveis de conforto mais elevados do que os perfis de "Família Consciente". Estes perfis foram adaptados para equipamentos atuais com base no Programa Brasileiro de Etiquetagem [PBE 2016]. O horário de uso dos equipamentos foi baseado na Pesquisa de Posse de Equipamentos e Hábitos de Uso do Procel [Eletrobras 2007]. A classificação de cargas para os perfis ficou da seguinte forma:

- Reguláveis - chuveiro, iluminação e aparelhos de ar condiciando;

- Flexíveis - máquina de lavar roupas;

- Dispensáveis - aspirador de pó e filtro de água;

- Indispensáveis - demais aparelhos elétricos.

A simulação modelou uma casa com três zonas térmicas: quarto de casal de $20 \mathrm{~m}^{2}$, quarto de solteiro $12 \mathrm{~m}^{2}$ e o restante da casa com $45 \mathrm{~m}^{2}$. As dimensões usadas para a casa são uma adaptação da casa simulada em [Lamberts et al. 2010b]. Os quartos foram projetados apenas para dimensionar o uso de energia dos aparelhos de ar-condicionado. Os dispositivos elétricos são atribuídos à zona térmica que representa o restante da casa. $\mathrm{O}$ arquivo de clima escolhido foi o da cidade de Niterói, disponibilizado no site do labEEE [INMET 2010]. A escolha do material utilizado na simulação e demais detalhes foram baseados em [Westphal and Lamberts 2006].

\section{Análise dos Resultados}

Foram executados 20 testes para cada padrão de consumo. O intuito foi modelar 20 dias diferentes, alterando-se o horário de funcionamento de cada dispositivo elétrico e as condições climáticas da simulação. 


\subsection{DEFLEGER-ECO}

Para comparar a eficiência do DEFLEGER-ECO, um dos mecanismos propostos em [Li and Zhang 2014] também foi simulado. Ele foi denominado aqui como GLD Comparativo. Este mecanismo apenas varia a temperatura do ar-condicionado de acordo com a variação do preço da energia. O objetivo do DEFLEGER-ECO é economizar, mas respeitando o nível de conforto do usuário.

Os quatro perfis de residência foram avaliados para verificar a economia durante o horário de pico, como mostra a Figura 5. Foi utilizada Intensidade 5, parâmetro explicado na Seção 4, e meta de percentual de economia de 30\%. Foi escolhido o percentual de economia $30 \%$, pois nem todos os perfis conseguiriam atingir essa economia. Desta forma pode-se analisar o comportamento do mecanismo quando a meta não é alcançada.

Nota-se que quanto maior o consumo de energia do perfil, maior a economia realizada pelo DEFLEGER-ECO. Os perfis da famílias "Conscientes" não conseguiram economizar o percentual de $30 \%$, respeitando os parâmetros de conforto do usuário, e foram enviadas mensagens notificando os clientes . Nestes perfis houve reagendamento de carga, mas mesmo assim a meta não foi alcançada. Os perfis das famílias "Sbanjas" alcançaram a meta de economia apenas com variação da Intensidade dos dispositivos Reguláveis. Em relação ao GLD Comparativo a quantidade de energia economizada pelo DEFLEGER-ECO foi muito superior.

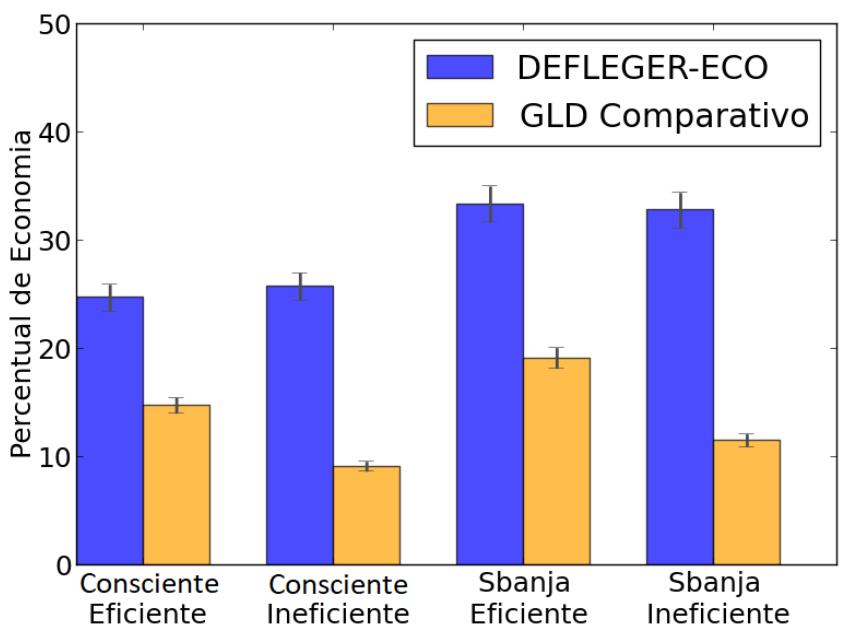

Figura 5. Eficiência dos mecanismos de GLD assumindo uma meta de $30 \%$ de economia durante o horário de pico.

Um segundo teste realizado foi a variação do percentual de economia configurado no gerenciador. O percentual de economia foi ajustado e alterado entre $10 \%$ até $90 \%$, usando perfil sbanja ineficiente e Intensidade 5, como mostra a Figura 6. Conforme aumenta-se o percentual de economia configurado, aumenta-se também a economia alcançada, até que o limite máximo seja atingido, ou seja, o limite dos parâmetros de conforto do usuário. Por isso, a economia em horário de pico fica próxima de $40 \%$ mesmo que a configuração seja de $90 \%$. Nos casos em que a meta de economia não é atingida, mensagens de alerta foram enviadas aos usuários. O DEFLEGER-ECO apresenta uma economia muito superior ao GLD Comparativo. É valido destacar que o mecanismo 
busca atingir o percentual de economia configurado, mas sem desrespeitar os parâmetros de conforto configurados pelo cliente, então quando a meta de economia não é alcançada o usuário é notificado.

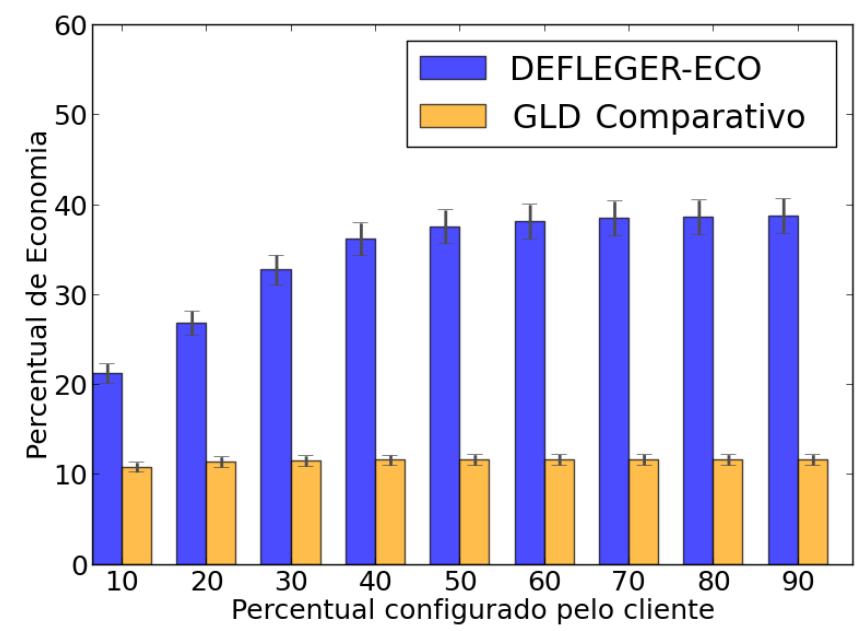

Figura 6. Eficiência dos mecanismos de GLD, variando-se a meta de economia durante o horário de pico.

\subsection{DEFLEGER-EMER}

O objetivo do DEFLEGER-EMER é garantir que a meta de redução seja alcançada durante eventos emergenciais. Os parâmetros de conforto do usuário são levados em consideração, mas a prioridade é alcançar a meta de redução.

Na análise do DEFLEGER-EMER, a meta de redução foi variada de $10 \%$ a $90 \%$, com intensidade 5 e perfil Sbanja Ineficiente. A Figura 7 mostra os resultados desta análise.

Neste teste, pode-se verificar a evolução dos grupos de redução de carga. Para redução de $10 \%$ e $30 \%$, a meta foi alcançada apenas com as reduções de potência dos dispositivos ajustáveis. Para 50\%, houve desligamento dos aparelhos de ar-condicionado e deslocamento de carga, que pode ser verificado com o aumento da demanda após o fim do período emergencial. Para $70 \%$ e $90 \%$, após a tentativa de redução de potência, verifica-se uma redução tão grande que o algoritmo é obrigado a desligar toda a casa e depois ir religando os aparelhos possíveis de serem religados, respeitando a meta de redução.

\section{Conclusão}

Os mecanismos de GLD, além de ajudarem no balanceamento entre a geração e a demanda de energia, também são uma excelente oportunidade para economizar energia ou tornar seu uso mais eficiente. O objetivo das propostas foi desenvolver mecanismos GLD voltados para o cenário brasileiro, sendo um de economia e outro emergencial. Observando-se os trabalhos relacionados, grande parte deles buscaram economizar através somente do ajuste de termostatos de aparelhos de ar-condicionado e aquecedores 

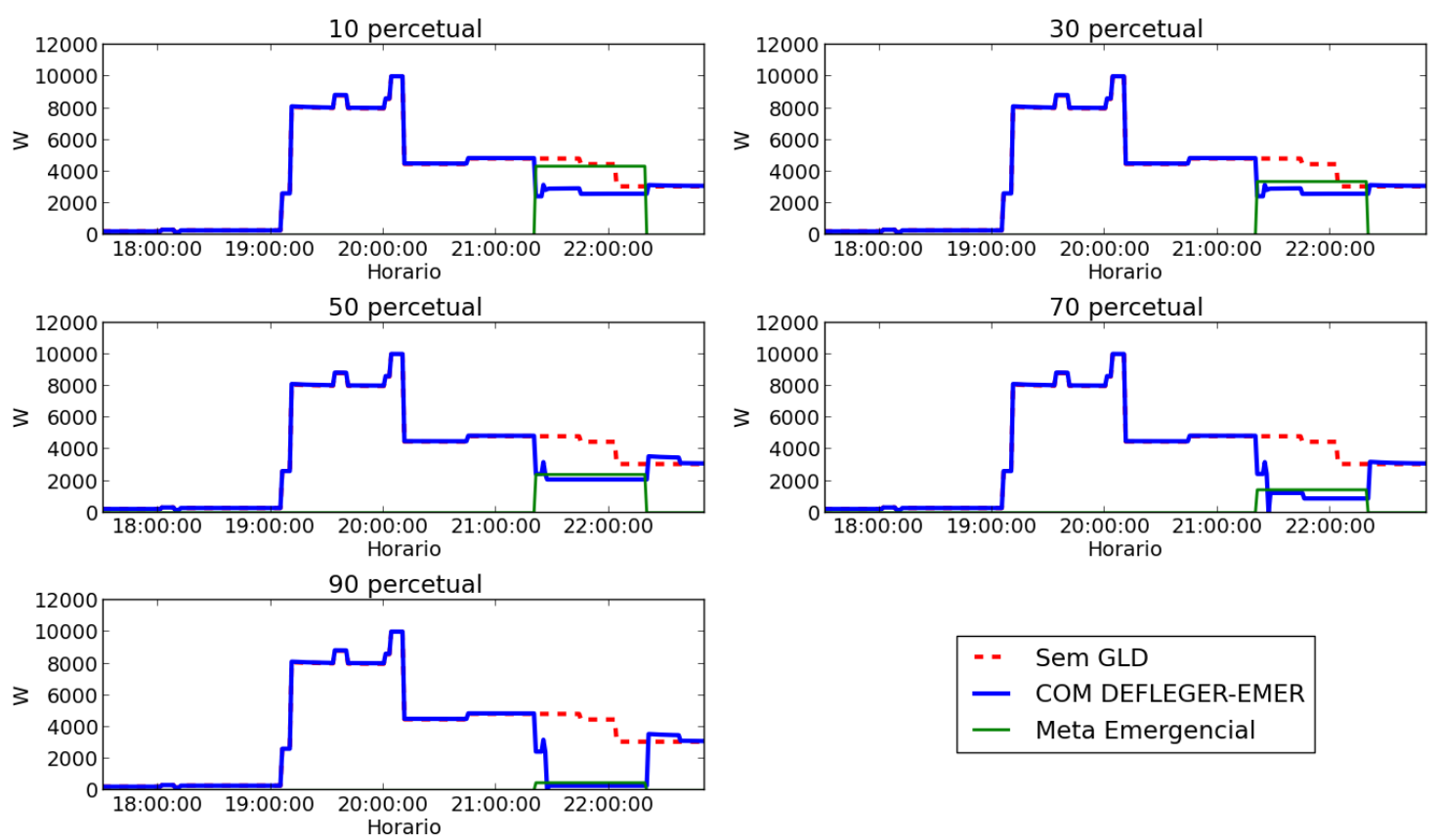

Figura 7. DEFLEGER-EMER - Gráfico de Demanda por Percentual.

ou somente através do agendamento de cargas. Então, foram propostos modelos que utilizam as duas estratégias ao mesmo tempo (ajuste de termostato e agendamento) e que, além disso, trabalham com a variação de potência dos dispositivos ajustáveis.

As propostas apresentaram facilidade de atender a diferentes perfis de usuários. Quanto menos dispositivos elétricos na residência, maior o peso que a energia gasta em iluminação tem na fatura. Ou seja, famílias com perfil mais simples podem economizar através do ajuste de potência da iluminação e dos chuveiros elétricos, não restringindo assim os programas apenas às casas com aparelhos de ar-condicionado. As propostas com agendamento já possibilitavam o atendimento de diferentes perfis de casa, entretanto é importante observar que a economia por agendamento traz apenas economia no horário de pico, pois a energia total consumida pelo usuário continua a mesma, uma vez que o dispositivo apenas foi reagendado para operar em outro horário.

O modelo de economia denominado DEFLEGER-ECO foi criado para economizar energia durante horário de pico da tarifa branca. O usuário configura os parâmetros de conforto e o percentual de energia que deve ser economizado. Se o percentual não for alcançado, um alerta é enviado ao usuário. Seu desempenho foi comparado ao mecanismo proposto no artigo [Li and Zhang 2014] e demonstrou grande potencial de economia, economizando até duas vezes mais.

O modelo emergencial, denominado DEFLEGER-EMER, visa reduzir a demanda durante eventos emergenciais. O mecanismo recebe os parâmetros de conforto configurados pelo usuário e aguarda a mensagem vinda da empresa de energia requisitando uma redução de carga. Ele procura atingir a meta de demanda respeitando o conforto do usuário. Entretanto, se necessário, os aparelhos elétricos serão desligados para alcançar a meta. Nos testes realizados, o DEFLEGER-EMER teve um bom desempenho, respeitando a meta estabelecida e atingindo a meta em até sete minutos. 


\section{Referências}

ANEEL (2020). Comparativo entre a tarifa branca e a tarifa convecional.

ANEEL-RN733 (2016). Resolução normativa $n^{0} 733$, de 6 de setembro de 2016. Estabelece as condições para a aplicação da modalidade tarifária horária branca.

Castro, T. B. (2016). Defleger: Deslocamento e flexibilidade de cargas no gerenciamento de energia residencial. Dissertação de M.Sc. Universidade Federal Fluminense.

Clearlyenergy (2016). https://www.clearlyenergy.com/residential-demand-responseprograms.

Conejo, A. J., Morales, J. M., and Baringo, L. (2010). Real-time demand response model. IEEE Transactions on Smart Grid, 1(3):236-242.

Eletrobras (2007). Pesquisa de posse de equipamentos e hábitos de uso: ano-base 2005, classe residencial. Relatório Brasil. Rio de Janeiro:ELETROBRAS/PROCEL.

EPE (2016). Resenha mensal do mercado de energia - número 103.

EPE (2019). Balanço energético nacional. Ministério de Minas e Energia.

Gelazanskas, L. and Gamage, K. A. (2014). Demand side management in smart grid: A review and proposals for future direction. Sustainable Cities and Society, 11:22-30.

INMET (2010). Arquivos climáticos inmet 2012.

Kinhekar, N., Padhy, N. P., and Gupta, H. O. (2013). Demand side management for residential consumers. In 2013 IEEE Power \& Energy Society General Meeting, pages $1-5$. IEEE.

Komninos, N., Philippou, E., and Pitsillides, A. (2014). Survey in smart grid and smart home security: Issues, challenges and countermeasures. IEEE Communications Surveys \& Tutorials, 16(4):1933-1954.

Lamberts, R., Ghisi, E., Pereira, C., and Batista, J. (2010a). Casa eficiente: consumo e geração de energia. Florianópolis: UFSC/LABEE, 2.

Lamberts, R., Ghisi, E., Pereira, C., and BATISTA, J. (2010b). Casa eficiente: simulação computacional do desempenho termo-energético. Florianópolis: UFSC/LabEEE, 4.

Lee, E.-K., Gadh, R., and Gerla, M. (2013). Energy service interface: Accessing to customer energy resources for smart grid interoperation. IEEE Journal on Selected Areas in Communications, 31(7):1195-1204.

Li, S. and Zhang, D. (2014). Developing smart and real-time demand response mechanism for residential energy consumers. In Power Systems Conference (PSC), 2014 Clemson University, pages 1-5. IEEE.

PBE (2016). Programa brasileiro de etiquetagem.

Tham, C.-K. and Zhou, C. (2013). Ambient sensing-based incentives for behavior modification in demand response. In Smart Grid Communications (SmartGridComm), 2013 IEEE International Conference on, pages 193-198. IEEE.

Westphal, F. and Lamberts, R. (2006). Introdução ao energyplus. Laboratório de Eficiência Energética em Edificações. 